\title{
openheart Aortic distensibility in Marfan syndrome: a potential predictor of aortic events?
}

\author{
Mitzi M van Andel (D) , ${ }^{1}$ Vivian de Waard, ${ }^{2}$ Janneke Timmermans, ${ }^{3}$ \\ Arthur J H A Scholte, ${ }^{4}$ Maarten P van den Berg, ${ }^{5}$ Aeilko H Zwinderman, ${ }^{6}$ \\ Barbara J M Mulder, ${ }^{1}$ Maarten Groenink ${ }^{1,7}$
}

\begin{abstract}
- Additional supplemental material is published online only. To view, please visit the journal online (http://dx.doi.org/10. 1136/openhrt-2021-001775)
\end{abstract}

To cite: van Andel MM, de Waard V, Timmermans J, et al. Aortic distensibility in Marfan syndrome: a potential predictor of aortic events?. Open Heart 2021;8: 001775. doi:10.1136/

openhrt-2021-001775

Received 13 July 2021 Accepted 23 September 2021

Check for updates

(C) Author(s) (or their employer(s)) 2021. Re-use permitted under CC BY-NC. No commercial re-use. See rights and permissions. Published by BMJ.

For numbered affiliations see end of article.

Correspondence to Dr Maarten Groenink; m. groenink@amsterdamumc.nl

\section{ABSTRACT}

Objectives Patients with Marfan syndrome (MFS) are prone to develop aortic aneurysms due to fragmentation of elastic fibres, resulting in reduced distensibility of the aorta. Reduced distensibility was previously shown to predict progressive descending aorta dilatation. Here, we investigated longitudinal changes in distensibility, as a potential predictor of aortic events.

Methods This retrospective study included all patients with MFS with at least four cardiac magnetic resonance examinations performed between 1996 and 2012. Aortic distensibility was assessed, in the ascending (level 1), proximal descending (level 2) and distal descending (level 3) aorta. Changes in distensibility were studied using linear mixed-effects regression models.

Results In total, 35 patients with MFS (age at inclusion 28 (IQR 23-32) years, 54\% men) were included. Mean aortic distensibility was already low (between $2.9 \times 10^{-3}$ / $\mathrm{mm} \mathrm{Hg} /$ year and $6.4 \times 10^{-3} / \mathrm{mm} \mathrm{Hg} /$ year) at all levels at baseline, and significantly decreased over time at levels 2 and 3 (respectively, $p=0.012$ and $p=0.002$ ). The rate of distensibility loss per year $\left(\times 10^{-3} / \mathrm{mm} \mathrm{Hg} /\right.$ year) was $0.01,0.03$ and $0.06 \times 10^{-3} / \mathrm{mm} \mathrm{Hg}$ at levels 1 , 2 and 3 , respectively. At inclusion, men exhibited very low distensibility, whereas women showed moderately reduced distensibility, gradually decreasing with age. Aortic dilatation rate at level 2 was associated with reduced aortic distensibility. However, we could not demonstrate a direct correlation between distensibility and clinical events during a follow-up of 22 years.

Conclusion Patients with MFS display reduced aortic distensibility already at an early age, inversely relating to aortic dilatation rate. However, in this selected patient group, distensibility seems less suitable as an individual predictor of aortic events.

\section{BACKGROUND}

Marfan syndrome (MFS) is a connective tissue disorder, due to mutations in the fibrillin-1 gene. Patient with MFS morbidity and mortality is mainly determined by progressive dilatation of the aorta, ultimately leading to potentially lethal aortic dissection when no timely surgical intervention is undertaken. Therefore, clinical management is directed

\section{Key questions}

What is already known about this subject?

- Patients with Marfan syndrome (MFS) may develop aortic dissection due to progressive dilatation in the thoracic aorta. Aortic distensibility has been shown to serve as a predictor for progressive descending thoracic aorta dilatation.

What does this study add?

- This retrospective study investigated longitudinal changes in aortic distensibility throughout the thoracic aorta by means of cardiac magnetic resonance in individual patients with MFS to assess aortic distensibility as a possible predictor of aortic events (aortic surgery or dissection).

How might this impact on clinical practice?

- Patients with MFS have low values of aortic distensibility already at an early age, inversely relating to aortic dilatation rate. However, distensibility seems less suitable as an individual predictor of aortic events.

at prophylactic aortic surgery, based on threshold values for aortic diameters. ${ }^{1}$ Due to aggressive surgical prophylactic management, life expectancy in patients with MFS has greatly improved in the past decades. ${ }^{2}$

Most aortic dilatations in MFS occur in the aortic root. Nevertheless, the more distal aorta may also be at risk for aortic dissection, especially after aortic root replacement. Proper timing of prophylactic surgery of the distal aorta appears to be more challenging in MFS, since aortic dissection often occurs at near normal diameters in these parts of the aorta after aortic root surgery. ${ }^{3}$ We argued that aortic distensibility could serve as a potential predictor of aortic events in these parts of the aorta, also taking into account that aortic root replacement itself might change distensibility in the more distal aorta. Aortic distensibility has already been widely investigated in different cohort studies with 
patients with MFS and associated with aortic complications, ${ }^{4-7}$ with thoracic aortic distensibility as the strongest predictor for progressive descending thoracic aorta dilatation. ${ }^{4}$ However, it is unknown how the rate of distensibility loss over time affects aortic events (aortic surgery or dissection) in individual patients with MFS.

The aim of this study was to investigate aortic distensibility as a predictor of aortic complications in patients with MFS over time. Furthermore, we compared rates of distensibility loss between men and women, and investigated the influence of medication use or aortic surgery on the rate of distensibility loss in individual patients with MFS.

\section{METHODS}

This study complies with the Declaration of Helsinki and was written in accordance with the Strengthening the Reporting of Observation Studies in Epidemiology statement. ${ }^{5}$ We intend to disseminate the main results to trial participants and will seek patient and public involvement in the development of an appropriate method of dissemination.

\section{Study design and participants}

All patients included in the current retrospective study were previously included in two studies performed earlier in the Amsterdam UMC-location AMC. The first study was conducted from 1996 to 2002. Herein, patients underwent two MRI scans with a median follow-up of 5.9 years. ${ }^{4}$ The second study was the COzaar in Marfan Patients Reduces aortic Enlargement (COMPARE) trial, ${ }^{6}$ a study that initially was conducted from 2008 to 2012, in which patients also underwent two MRI scans with a median follow-up of 3.1 years between scans. At time of inclusion, patients were not involved in the design, management or conduct of the trial. Only patients who underwent all four MRI scans were included in the current study.

\section{Data sources and measurement}

Baseline patient characteristics were extracted from the original study database of 1996. Additional information on medication use and events during follow-up of the trial were extracted from the COMPARE database. Follow-up data concerning elective aortic root replacement, aortic dissection and death were available until 2018, and were extracted from the extended COMPARE trial database. ${ }^{7}$

\section{Cardiac magnetic resonance}

All cardiac magnetic resonance (CMR) scans were performed with a 1.5 Tesla MR system (either Philips ACS-NT15, Philips Medical Systems, Best, the Netherlands, or Magnetom Vision or Avanto, Siemens Medical Systems, Erlangen, Germany).

In all scans, commercially available gadoliniumenhanced spoiled gradient echo techniques were used to visualise the entire aorta. Exact MRI protocol of both studies concerning the ECG-gated cine MRI has been described elsewhere earlier. ${ }^{4}$ In brief, high resolution

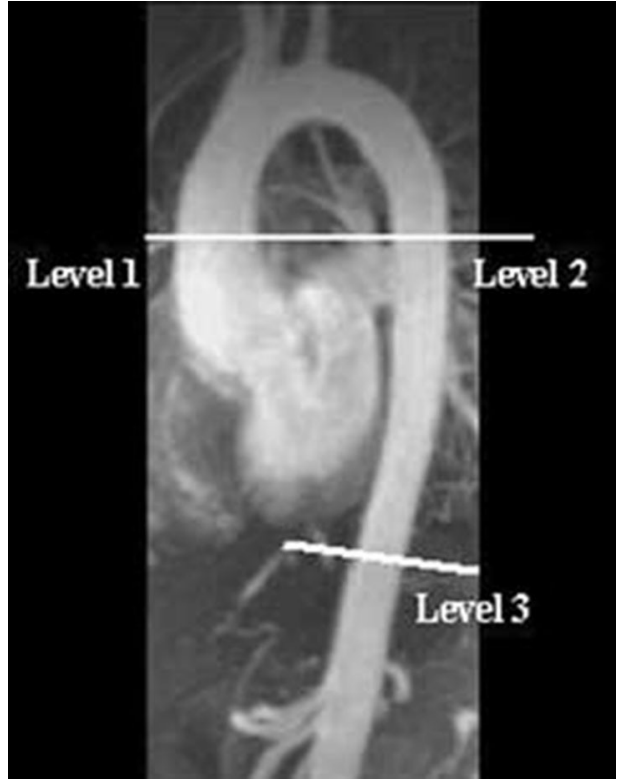

Figure 1 The aorta of a patient with Marfan syndrome. At indicated levels, aortic distensibility was measured. Level 1 (D1)=ascending aorta, Level 2 (D2)=proximal descending aorta, Level 3 (D3)=distal descending aorta.

gradient echo pulse sequences were applied perpendicular to the aorta at three levels as indicated in figure 1; (1) ascending aorta (D1), (2) descending thoracic aorta at the level of the bifurcation of the pulmonary artery (D2) and (3) descending aorta at the level of the diaphragm (D3). During the MRI scans, blood pressure was measured by a sphygmomanometer cuff.

\section{Aortic distensibility}

Syngo.via image analysis software (Siemens, Erlangen, Germany) was used for image analysis. Aortic distensibility was re-measured and re-calculated per patient, on all four scans at all three levels, by a single analyst (MMvA) using the previously conducted cine MRI and non-invasively measured blood pressure during CMR, as described by Groenink et al. ${ }^{9}$ The re-measured distensibility values are in accordance with the values calculated in the original studies (figure 2). Bland-Altman analysis at level 2 showed no significant difference $(\mathrm{p}=0.304)$, meaning no proportional bias.

Aortic contours were drawn manually on the modulus images of all cardiac phases. Aortic distensibility was calculated according to the following formula:

$$
\mathrm{D}=\left(\left(\mathrm{A}_{\max }-\mathrm{A}_{\min }\right) / \mathrm{A}_{\min }\right) / \text { pulse pressure }
$$

where $\mathrm{D}=$ distensibility $(/ \mathrm{mm} \quad \mathrm{Hg}), \quad \mathrm{A}_{\max }=$ maximal (systolic) aortic area $\left(\mathrm{mm}^{2}\right), \mathrm{A}_{\min }=$ minimal (diastolic) aortic area $\left(\mathrm{mm}^{2}\right)$, pulse pressure $=$ systolic blood pressure-diastolic blood pressure ( $\mathrm{mm} \mathrm{Hg}$ ).

\section{Statistical analysis}

Statistical analyses were performed using R V.1.0.153 (RStudio Team, Boston, Massachusetts, USA) and SPSS V.22 (IBM, Armonk, New York, USA). Data are 


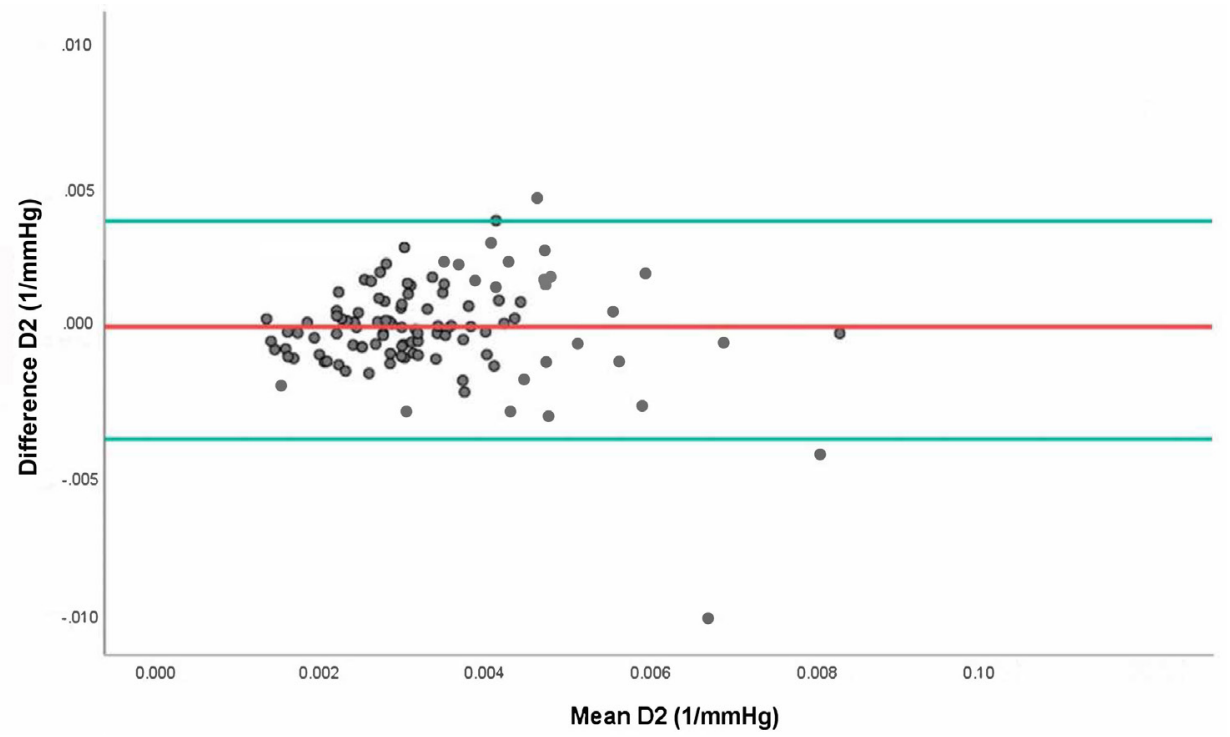

Figure 2 Bland-Altman analysis of distensibility values at the level of the thoracic proximal descending aorta. Mean $0.0022 \pm 0.0019$, upper: 0.0039 , lower: -0.0037 .

summarised as $\mathrm{n}(\%)$, mean $\pm \mathrm{SD}$ and median (IQR). $\mathrm{P}$ values of $<0.05$ were considered statistically significant.

Distensibility at all levels was analysed using linear mixed-effects regression models. Spline transformations were used to find the best fit of the regression line of distensibility on time and age. Likelihood-ratio tests were used to compare fit of different models. Finally, linear regression lines were found to best fit the distensibility data.

Additional fixed-effects were sex, medication use and aortic root surgery as time-dependent covariates, as both main-effects and their interactions with age. Change in rate of distensibility loss before and after surgery and before and after start of medication use was evaluated with a change-point model.

\section{RESULTS}

\section{Study population}

In total, 78 adult patients with MFS participated in the first study (1996-2002). Of these 78 patients, 41 patients were also included in the COMPARE trial. Imaging and blood pressure measurements on all four moments in time were available in 35 patients. Aortic distensibility was measured and calculated at all three levels. The baseline characteristics are shown in table 1. Included patients were adults ( $\geq 18$ years) diagnosed with MFS according to the Ghent criteria of $1996 .{ }^{10}$ Aortic dimension was significantly higher in men than in women at all levels. Aortic root diameters in men are at baseline already near to dimenstion indicative for elective aortic surgery. Table 2

\section{Table 1 Baseline characteristics}

\begin{tabular}{|c|c|c|c|c|}
\hline & \multirow{2}{*}{$\begin{array}{l}\text { All patients } \\
n=35\end{array}$} & \multirow{2}{*}{$\begin{array}{l}\text { Women } \\
n=16\end{array}$} & \multirow{2}{*}{$\begin{array}{l}\text { Men } \\
n=19\end{array}$} & \multirow[b]{2}{*}{$P$ value } \\
\hline & & & & \\
\hline \multicolumn{5}{|l|}{ Patient characteristics } \\
\hline Age at inclusion, years & $28(23-32)$ & $30(24-32)$ & $27(21-36)$ & 0.051 \\
\hline Body surface area, $\mathrm{m}^{2}$ & $2.08 \pm 0.22$ & $1.96 \pm 0.18$ & $2.19 \pm 0.20$ & $<0.001$ \\
\hline Systolic blood pressure & $116 \pm 10.4$ & $116 \pm 10.8$ & $116 \pm 9.9$ & 0.368 \\
\hline Diastolic blood pressure & $60 \pm 10.5$ & $61 \pm 10.4$ & $60 \pm 10.5$ & 0.312 \\
\hline Mean arterial pressure & $79 \pm 9.6$ & $79 \pm 10.0$ & $78 \pm 9.0$ & 1.000 \\
\hline$\beta$-blocker (since 1996) & $28(80 \%)$ & $11(69 \%)$ & 17 (89\%) & 0.001 \\
\hline Losartan (since 2008) & $25(71 \%)$ & $11(69 \%)$ & $14(74 \%)$ & 0.505 \\
\hline \multicolumn{5}{|l|}{ Aortic dimension by MRI } \\
\hline Aortic root (mm) & $42(40-45)$ & $40(36-44)$ & $45(41-46)$ & $<0.001$ \\
\hline Ascending aorta (mm)—Level 1 & $29(23-35)$ & $28(23-35)$ & $29(24-35)$ & 0.040 \\
\hline Proximal descending aorta (mm)—Level 2 & $24(17-35)$ & $23(17-25)$ & $25(21-35)$ & $<0.001$ \\
\hline Distal descending aorta $(\mathrm{mm})$ _Level 3 & $21(17-28)$ & $21(17-25)$ & $22(19-28)$ & $<0.001$ \\
\hline
\end{tabular}


Table 2 Distensibility $\left(\times 10^{-3} / \mathrm{mm} \mathrm{Hg}\right)$ at baseline and at the last scan in 2012

\begin{tabular}{lll}
\hline & $\mathbf{1 9 9 6}$ & $\mathbf{2 0 1 2}$ \\
\hline D1 & $2.92 \pm 1.16$ & $2.92 \pm 1.22$ \\
D2 & $3.76 \pm 1.59$ & $2.85 \pm 1.07$ \\
D3 & $6.39 \pm 2.07$ & $4.76 \pm 2.07$ \\
\hline
\end{tabular}

shows distensibility values at baseline and at the last scan in 2012. At baseline (1996), none of the 35 patients included in the current study had a history of aortic surgery. At follow-up in 2018 (22 years), prophylactic aortic root surgery had been performed in 19 patients $(54 \%)$. Three patients had experienced a type B aortic dissection (men 2 vs women $1, \mathrm{p}=0.266$ ) after previous elective aortic root surgery.

\section{Changes in distensibility over time}

Aortic distensibility at all levels decreased approximately linearly with age (figure 3 ). This decrease was significant at level $2(\mathrm{p}=0.012)$ and level $3(\mathrm{p}=0.002)$. The annual decline in distensibility was $0.01,0.03$ and $0.06 \times 10^{-3} /$ $\mathrm{mm} \mathrm{Hg}$ (all with SE 0.02) on levels 1, 2 and 3, respectively. These results remained significant after correction for blood pressure. Aortic dilatation rate at level 2 $(0.10 \pm 0.14 \mathrm{~mm} /$ year $)$ was associated with reduced aortic distensibility.

\section{Men versus women}

At baseline, distensibilty was higher in women at all levels throughout the aorta when compared with men, and it deteriorated over time (table 3, figure 4). Men had already low distensibility at inclusion, without further decline over time. The differences between men and women in the magnitude of decline at levels 1 and 3 were significant (men: 0.010 and women: $-0.042, \mathrm{p}=0.029$; men: -0.033 and women: $-0.11, p=0.038$, respectively). This is in accordance with healthy controls, where women also

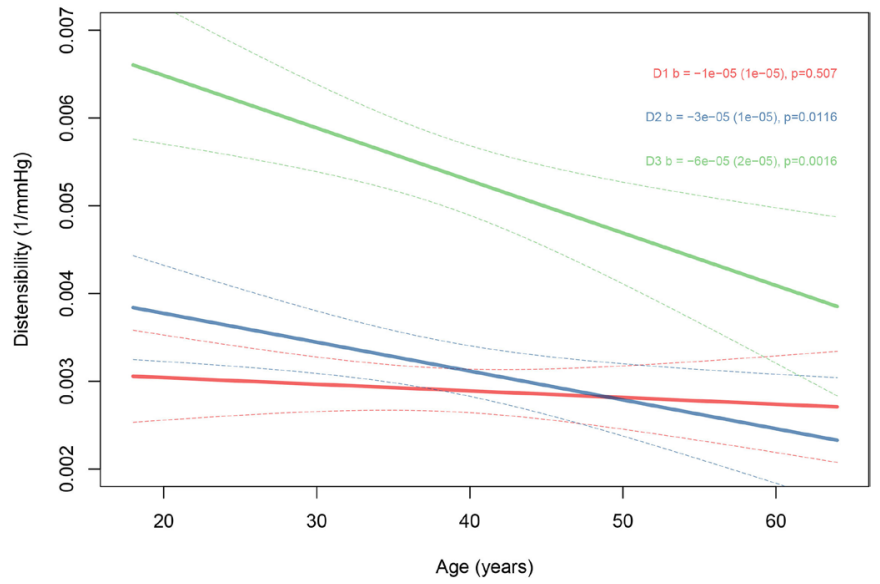

Figure 3 Linear regression lines of distensibility at all three levels in all patients $(n=35)$ decrease with age. The decrease is statistically significant at D2 and D3. Error bars: Cl 95\% of the regression line.
Table 3 Associations between distensibility loss per year and sex and medication use. Differences in distensibility loss per year: slope $(\mathrm{SE})\left(\times 10^{-3} / \mathrm{mm} \mathrm{Hg}\right)$

\begin{tabular}{llll}
\hline \multicolumn{4}{l}{ All patients $(\mathbf{n}=\mathbf{3 5})$} \\
\cline { 2 - 4 } & $\begin{array}{l}\text { Male versus } \\
\text { female }\end{array}$ & $\begin{array}{l}\text { Beta-blocking: } \\
\text { yes versus no }\end{array}$ & $\begin{array}{l}\text { Losartan: yes } \\
\text { versus no }\end{array}$ \\
\hline D1 & $0.052(0.024)$ & $0.017(0.032)$ & $-0.033(0.023)$ \\
& $p=0.029$ & $p=0.053$ & $p=0.30$ \\
D2 & $0.049(0.027)$ & $0.015(0.034)$ & $0.017(0.026)$ \\
& $p=0.071$ & $p=0.44$ & $p=0.81$ \\
D3 & $0.077(0.037)$ & $0.037(0.051)$ & $-0.018(0.037)$ \\
& $p=0.038$ & $p=0.72$ & $p=0.81$ \\
\hline
\end{tabular}

show higher distensibility than age-matched men early in life. ${ }^{11}$ This discrepancy in distensibility between sexes is diminished after 50 years of age at all levels within the aorta when distensibility is reduced to a minimum.

In comparison to values from healthy controls described in the literature, men with MFS in our cohort showed the same rate of distensibility loss as control individuals at level $2^{12}\left(-0.033\right.$ vs $-0.036 \times 10^{-3} / \mathrm{mm} \mathrm{Hg} /$ year $)$, while in women with MFS distensibility deteriorated here faster than in controls $\left(-0.11\right.$ vs $-0.05 \times 10^{-3} / \mathrm{mm} \mathrm{Hg} /$ year $)$ at this level.

Moreover, men with MFS underwent aortic root surgery at significantly earlier age than women in our cohort (men 37 year vs women 44 year, $\mathrm{p}<0.001$ ). At inclusion, aortic root dimensions in men were larger than in women (men $45 \mathrm{~mm}$ vs women $40 \mathrm{~mm}, \mathrm{p}<0.001$ ) (table 1 ).

\section{Native aortic root versus previous aortic root surgery}

During follow-up until 2018, 19 of the 35 patients (54\%) underwent prophylactic aortic root surgery. We anticipated changes in distal aortic distensibility in the operated patients, by the haemodynamic influence of a stiff vascular graft. ${ }^{13}$ However, we could not demonstrate significant differences in rate of distensibility loss with age between patients with a native aortic root during
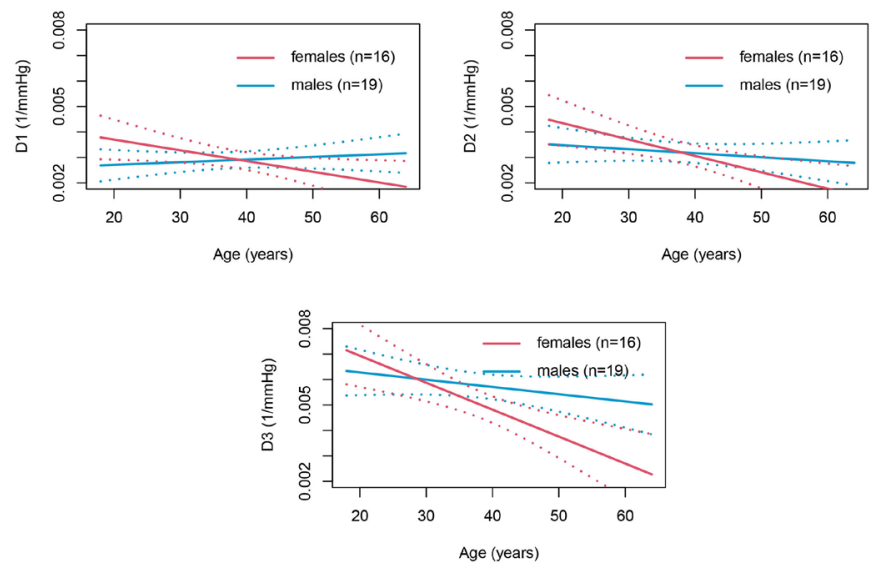

Figure 4 Linear regression lines of distensibility in men $(n=19)$ and women $(n=16)$ at all levels decrease with age. Error bars: $\mathrm{Cl}$ 95\% of the regression lines. 

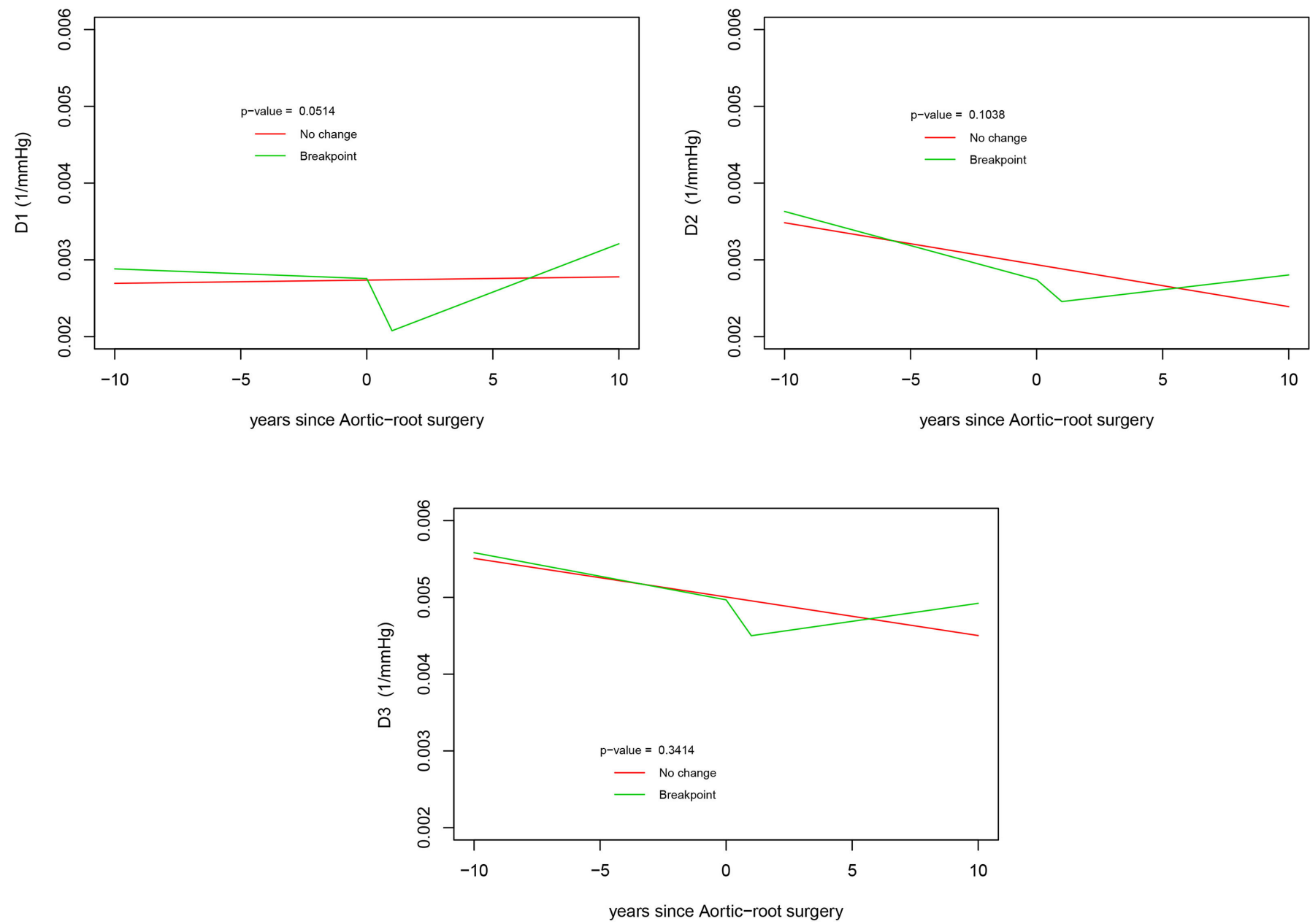

Figure 5 Change in rate of distensibility loss before and after surgery was evaluated with a change-point model. Figure 5 shows distensibility over time in the 19 patients that have had aortic root surgery at some point in time (time $0=$ time of surgery=breakpoint). Red line=regression line of distensibility over time. Green line=two regression lines connected (breakpoint) at point of surgery (before and after surgery). There is no evidence for aortic root surgery being a breakingpoint for aortic distensibility loss in time.

follow-up and patients who had undergone prophylactic aortic root surgery $(\mathrm{p}=0.20)$. Furthermore, we could not demonstrate a statistically significant difference in rate of change in distensibility before and after surgery in operated patients on, respectively, levels 1,2 and 3 ( $\mathrm{p}=0.0514$, $\mathrm{p}=0.1038, \mathrm{p}=0.3414$ ) (figure 5). Although, there exists no significant evidence for a breakpoint at time of aortic root surgery in the change of distensibility, figure 5 shows a trend towards an increasing distensibility after aortic root surgery at all levels (green line).

Patients with MFS mostly use medication blocking the $\beta$-adrenergic receptors or the angiotensin-II receptor type 1 to reduce blood pressure. Therefore, we assessed if these drugs influenced aortic distensibility. The use of either $\beta$-blocking agents or losartan (online supplemental figures 1 and 2) had no significant effect on the rate of change in distensibility. Only patients using $\beta$-blocker showed a trend towards a decreased rate of distensibility loss at level 1 compared with patients not using $\beta$-blockers $(\mathrm{p}=0.053) \quad($ table 3$)$.

\section{DISCUSSION}

Our study showed low distensibility at all levels in the thoracic aorta of young patients with MFS, which decreased over time. Men with MFS had lower distensibility than women with MFS at baseline. However, distensibility was stable in men, suggesting that aortic stiffening had taken place prior to the age of 27 years. In contrast, in women with MFS, the aortic distensibility deteriorated later in life compared with men. Moreover, men with MFS underwent aortic root surgery at significantly younger age than women. However, rate of distensibility loss did not correlate significantly with prophylactic aortic root surgery or medication use. Aortic dissection occurred in three patients, and only after aortic root surgery.

A number of studies have measured aortic distensibility in healthy subjects. ${ }^{11} 1214$ Although it is of great importance that these values have been established and we made an attempt to compare these to our cohort, it remains difficult to reliable compare them to our population with MFS. One study of healthy controls comprised 
children and young adults up to the age of 30 years. ${ }^{14}$ This cohort does not overlap with our MFS cohort, since the mean age of our patients with MFS was already 28 years at inclusion. Another study was conducted in an Asian population, which makes it hard to compare, because significant differences in clinical features of the cardiovascular system have been demonstrated between a Caucasian and Asian population with MFS. ${ }^{15}$ Therefore, we used the distensibility measurements in healthy individuals (age 21-60 years) of Aquero et $a l^{12}$ as reference for our study to evaluate the changes in distensibility in relation to our population with MFS.

Overall, distensibility at relatively young age appears to be lower in patients with MFS than in healthy controls, which applies to both sexes. Indeed, men with MFS at an average age of 27 showed an aortic distensibility of 2.96 in the ascending aorta and 3.31 in the descending aorta in our study, whereas in healthy men, aortic distensibility was 7.0 in the ascending aorta and 5.2 in the descending aorta between the ages of 21 and 30 years. ${ }^{12}$ In women with MFS at an average age of 30, an aortic distensibility of 3.31 in the ascending aorta and 3.42 in the descending aorta was measured, which was also lower compared with healthy women, where aortic distensibility was 8.4 in the ascending aorta and 6.0 in the descending aorta. However, at an age of $>50$ years, our patients with MFS and healthy controls have a similarly low distensibility in ascending and descending aorta (approximately around 4.0 in men and women), which raises the question what the prognostic value is of measuring aortic stiffness in older patients with MFS.

In men, the severe reduction in distensibility before the age of 30, seems indicative for excessive loss of aortic elastic integrity, perhaps explaining why men were operated at younger age than women. The decision to operate is based on aortic root diameters, not aortic distensibility. Many of the men in our study already reached the established tresholds for aortic root surgery at inclusion, and it would thus have been of interest to know the rate of distensibility loss between age 15 and 30 years to evaluate if that could have predicted aortic root surgery, before clear changes in aortic diameters.

However, lower aortic distensibility beyond the aortic root fits the concept that this precedes dilation. Prophylactic aortic root surgery is a predictor for type B dissection in patients with MFS, ${ }^{3}$ which could be a reason that more often type B dissections occur in men at younger age. In our study, however, lower distensibility did not correlate with aortic root surgery, possible implying that these patients are more seriously affected from the start.

\section{METHODOLOGIC CONSIDERATIONS}

The limitations of the study include its retrospective design and the relatively small patient number that was used. In addition, an age-matched control group would have provided control data to compare to other studies with healthy individuals.
Measurements of aortic diameters, distensibility and pulse wave velocity have been shown to be reproducible, as described in a previous report. ${ }^{16}$ However, to use longitudinal measurements of aortic distensibility reproducibly, a number of influencial factors need to be controlled. Aortic distensibility is blood-pressure dependent. However, correction for blood pressure did not change our results. Although blood pressure is measured during the scan, it was not continuous. Moreover, drawing the aortic contours on the modulus images remains manual labour that may vary between different analysts. Additionally, aortic extension has shown to be asymmetric in undiseased aortas of young and healthy volunteers, ${ }^{17}$ which makes it even harder to evaluate distensibility on measurements taken only on certain levels of the aorta. All these factors complicate to measure distensibility on a routine basis. Thereby, our population with MFS was relatively healthy, concerning aortic surgery or dissection in their history, due to inclusion criteria for the clinical studies wherein they participated. Therefore, it would still be of interest to study a more severely affected adolescent population with MFS to observe especially in men with MFS, when the decline in aortic distensibility occurs. In this moment, we would, however, not recommend values of aortic distensibilty as a valid predictor of aortic events.

\section{CONCLUSION}

Patients with MFS have low distensibility at all levels of the aorta at young age, which decreased over time. In our study, men showed lower distensibility and larger aorta's at a younger age than women. In addition, men underwent aortic root surgery at younger age. However, no change in distensibility rates was observed before and after surgery. Therefore, aortic distensibility do not appear to be a suitable predictor of aortic events.

\section{Author affiliations \\ ${ }^{1}$ Cardiology, Amsterdam UMC - Location AMC, Amsterdam, The Netherlands ${ }^{2}$ Medical Biochemistry, Amsterdam UMC - Locatie AMC, Amsterdam, The Netherlands \\ ${ }^{3}$ Cardiology, Radboud University Hospital, Nijmegen, The Netherlands ${ }^{4}$ Cardiology, Leiden University Medical Center, Leiden, The Netherlands ${ }^{5}$ Cardiology, University Medical Center Groningen, Groningen, The Netherlands ${ }^{6}$ Clinical Epidemiology, Biostatistics and Bioinformatics, Amsterdam UMC - Locatie AMC, Amsterdam, The Netherlands \\ ${ }^{7}$ Radiology, Amsterdam UMC - Location AMC, Amsterdam, The Netherlands}

Contributors AHZ, BM and MG proposed and initiated the COMPARE trial. MMvA, $M G$ and BM defined the research strategy of the current study. AHZ provided statistical expertise. AS, MvdB and JT are our collaborators. MMvA, MG and BM drafted the manuscript. AHZ and VdW revised the manuscript for important intellectual content. All authors read and approved the final manuscript.

Funding This work was supported by private funding via the AMC Foundation.

Competing interests VdW obtained grants from the AMC Foundation, supporting MMvA during the conduct of the study.

Patient consent for publication Consent obtained directly from patient(s).

Ethics approval The COMPARE trial was conducted with approval of the Medical Ethical Committee of the Amsterdam UMC-location AMC. Written informed consent was obtained from all participants.

Provenance and peer review Not commissioned; externally peer reviewed. 
Data availability statement Data are available upon reasonable request. The data underlying this article will be shared on reasonable request to the corresponding author.

Open access This is an open access article distributed in accordance with the Creative Commons Attribution Non Commercial (CC BY-NC 4.0) license, which permits others to distribute, remix, adapt, build upon this work non-commercially, and license their derivative works on different terms, provided the original work is properly cited, appropriate credit is given, any changes made indicated, and the use is non-commercial. See: http://creativecommons.org/licenses/by-nc/4.0/.

ORCID iD

Mitzi M van Andel http://orcid.org/0000-0002-4629-568X

\section{REFERENCES}

1 Baumgartner H, De Backer J, Babu-Narayan SV, et al. 2020 ESC guidelines for the management of adult congenital heart disease. Eur Heart J 2021;42:563-645.

2 Silverman DI, Burton KJ, Gray J, et al. Life expectancy in the Marfan syndrome. Am J Cardiol 1995;75:157-60.

3 den Hartog AW, Franken R, Zwinderman AH, et al. The risk for type B aortic dissection in Marfan syndrome. J Am Coll Cardiol 2015;65:246-54.

4 Nollen GJ, Groenink M, Tijssen JGP, et al. Aortic stiffness and diameter predict progressive aortic dilatation in patients with Marfan syndrome. Eur Heart J 2004;25:1146-52.

5 von Elm E, Altman DG, Egger M, et al. The strengthening the reporting of observational studies in epidemiology (STROBE) statement: guidelines for reporting observational studies. Lancet 2007;370:1453-7.

6 Groenink M, den Hartog AW, Franken R, et al. Losartan reduces aortic dilatation rate in adults with Marfan syndrome: a randomized controlled trial. Eur Heart J 2013;34:3491-500.
7 van Andel MM, Indrakusuma R, Jalalzadeh $\mathrm{H}$, et al. Long-term clinical outcomes of losartan in patients with Marfan syndrome: follow-up of the multicentre randomized controlled compare trial. Eur Heart J 2020;41:4181-7.

8 den Hartog AW, Franken R, de Witte P, et al. Aortic disease in patients with Marfan syndrome: aortic volume assessment for surveillance. Radiology 2013;269:370-7.

9 Groenink M, de Roos A, Mulder BJ, et al. Changes in aortic distensibility and pulse wave velocity assessed with magnetic resonance imaging following beta-blocker therapy in the Marfan syndrome. Am J Cardiol 1998;82:203-8.

10 De Paepe A, Devereux RB, Dietz HC, et al. Revised diagnostic criteria for the Marfan syndrome. Am J Med Genet 1996;62:417-26.

11 Kim EK, Chang S-A, Jang SY, et al. Assessment of regional aortic stiffness with cardiac magnetic resonance imaging in a healthy Asian population. Int J Cardiovasc Imaging 2013;29 Suppl 1:57-64.

12 Aquaro GD, Cagnolo A, Tiwari KK, et al. Age-dependent changes in elastic properties of thoracic aorta evaluated by magnetic resonance in normal subjects. Interact Cardiovasc Thorac Surg 2013;17:674-9.

13 Lejay A, Geny B, Kolh P, et al. Effects of aortic graft implantation on heart and downstream vessels: an artery is not a rigid pipe. Eur $J$ Vasc Endovasc Surg 2019;58:477-8.

14 Voges I, Jerosch-Herold M, Hedderich J, et al. Normal values of aortic dimensions, distensibility, and pulse wave velocity in children and young adults: a cross-sectional study. J Cardiovasc Magn Reson 2012;14:77.

15 Franken R, den Hartog AW, van de Riet L, et al. Clinical features differ substantially between Caucasian and Asian populations of Marfan syndrome. Circ J 2013;77:2793-8.

16 Groenink M, de Roos A, Mulder BJ, et al. Biophysical properties of the normal-sized aorta in patients with Marfan syndrome: evaluation with Mr flow mapping. Radiology 2001;219:535-40.

17 van Prehn J, Vincken KL, Sprinkhuizen SM, et al. Aortic pulsatile distention in young healthy volunteers is asymmetric: analysis with ECG-gated MRI. Eur J Vasc Endovasc Surg 2009;37:168-74. 